\title{
Constructal Design aplicado à otimização geométrica de um material de alta condutividade térmica em forma de "T"
}

\author{
Constructal Design applied to geometric optimization of a material \\ with high thermal conductivity
}

\author{
M. K. Rodrigues ${ }^{1} *$; M. M. Goulart ${ }^{1}$; D. Helbig ${ }^{2,4}$; G. M. C. Magalhães ${ }^{1}$; \\ I. C. A. Junior ${ }^{3}$; L. A. O. Rocha ${ }^{1,2}$; L. A. Isoldi ${ }^{1,4}$; E. D. dos Santos ${ }^{1,4}$ \\ ${ }^{I}$ Programa de Pós-Graduação em Modelagem Computacional (PPGMC), Universidade Federal do Rio Grande \\ (FURG), 96.203-900, Rio Grande-RS, Brasil \\ ${ }^{2}$ Departamento de Engenharia Mecânica (DEMEC), Universidade Federal do Rio Grande do Sul (UFRGS), \\ 90.050-170, Porto Alegre-RS, Brasil \\ ${ }^{3}$ Instituto Federal de Educação, Ciência e Tecnologia do Rio Grande do Sul (IFRS), 96.201-460, \\ Rio Grande-RS, Brasil \\ ${ }^{4}$ Escola de Engenharia (EE), Universidade Federal do Rio Grande (FURG), 96.203-900, Rio Grande-RS, Brasil
}

*michel.professor@yahoo.com.br

(Recebido em 17 de outubro de 2014; aceito em 07 de março de 2015)

\begin{abstract}
O presente trabalho utiliza o método Constructal Design no estudo numérico da configuração geométrica de um material de alta condutividade térmica em forma de "T" que minimiza a resistência ao fluxo de calor, uma vez que as áreas ocupadas pelos materiais de alta e baixa condutividade são mantidas constantes. Para a solução numérica da equação da difusão do calor foi utilizado o método de elementos finitos (FEM), mais especificamente a ferramenta PDETOOL (Partial Differential Equations Tool) do software MATLAB. O objetivo deste trabalho é a minimização da resistência térmica do sistema gerador de calor, com baixa condutividade térmica, através da inserção de uma via em formato de "T", cujo material possui alta condutividade térmica e volume constante, sendo variáveis as dimensões correspondentes ao ramos simples e bifurcados. A geometria ótima deste material foi aquela que conduziu para a menor resistência térmica. Os resultados mostram que a geometria ótima conduz a uma diminuição da temperatura máxima mínima em aproximadamente $33 \%$ em relação a uma geometria não otimizada. Finalmente, o presente trabalho mostrou que a geometria ótima é aquela que melhor distribui as imperfeições, ou seja, os pontos quentes (pontos de temperatura máxima), o que vai ao encontro do princípio Constructal da ótima distribuição das imperfeições.
\end{abstract}

Palavras-chave: Constructal Design, otimização geométrica, material de alta condutividade térmica.

This work uses the Constructal Design method in the numerical study of geometric configuration of a Tshaped material of high thermal conductivity which minimizes the resistance of heat flux, since the areas occupied by the materials of high and low conductivity are kept constant. To solve numerically the heat diffusion equation it is used the finite element method (FEM), more specifically the PDETOOL of MATLAB. The objective is to minimize the thermal resistance of the heat generating system, with low thermal conductivity, by inserting a T-shaped pathway, whose material has high thermal conductivity and constant volume, being the dimensions of single and bifurcated branches free to vary. The optimal geometry of this material was that which led to lower thermal resistance. The results showed that the optimal geometry leads to a decrease of the minimum dimensionless maximum temperature in about $33 \%$ in comparison with a non-optimized geometry. Finally, this study showed that the optimum geometry is the one that best distributes imperfections, i.e., the hot spots (points of maximum temperature), which is in agreement with the Constructal principle of optimal distribution of imperfection.

Keywords: Constructal Design, geometric optimization, high thermal conductivity material.

\section{INTRODUÇÃO}

O aprimoramento crescente da tecnologia, da indústria moderna e a importância no aperfeiçoamento das técnicas de transferência do calor ganhou espaço significativo no desenvolvimento da engenharia nas áreas da eletromecânica, eletroeletrônica e biomecânica.

Especificamente no campo da tecnologia dos equipamentos eletrônicos a densidade do fluxo de calor de chips aumentou muito devido à minimização do volume ocupado por estes materiais 
com uma mesma capacidade de processamento e geração de energia. Consequentemente, o desempenho global do sistema, bem como a segurança do mesmo, são significativamente afetados pela ventilação e refrigeração dos dispositivos eletrônicos. Assim, os estudos no campo do resfriamento de dispositivos eletrônicos tornaram-se essenciais à evolução de materiais e circuitos eletrônicos com escalas cada vez menores, mantendo um grande desempenho.

Dessa forma, o resfriamento do sistema eletrônico tornou-se uma importante questão que motiva à engenharia no desenvolvimento de mecanismos de transferência do calor, de novas ligas metálicas e a da otimização da geometria destes materiais. Nos sólidos, os materiais de alta condutividade térmica possibilitam melhores condições à transferência do calor por difusão térmica de forma autônoma e rápida [1,2].

Em [3] é fornecido um modelo geral para determinar a resistência térmica dos caminhos de alta condutividade distribuídos em um material com geração de calor e de configuração geométrica arbitrária. A resistência térmica do conjunto é obtida através de uma soma ponderada de todas as resistências térmicas. Os resultados mostram que para diferentes configurações geométricas o método utilizado se mostra consistente em relação às soluções analíticas e numéricas disponíveis.

Posteriormente, em [4] é realizado o estudo sobre vias de alta condutividade em forma de "Y" inseridas em um sólido de forma retangular com baixa condutividade térmica. A geometria da via em formato de "Y" foi avaliada empregando-se Constructal Design. Os resultados indicaram que a geometria ótima obtida para a via em formato de "Y" conduziu a um desempenho aproximadamente $46 \%$ melhor do que o obtido com a melhor forma geométrica de uma via em formato de "I".

No trabalho de [5] foi aplicado Constructal Design para descobrir a configuração que facilita a troca térmica através de vias em formas de " $\mathrm{X}$ " de um material de alta condutividade térmica incorporado em um solido retangular com geração de calor de baixa condutividade térmica. $\mathrm{O}$ objetivo do referido trabalho foi de minimizar a temperatura máxima em excesso de todo o sistema, independentemente do local em que estão localizados. Os resultados mostraram que, dentre outras informações, as vias condutoras em " $\mathrm{X}$ " chega a ser mais do que 50\% superior à troca térmica, quando comparados com configurações em forma de "I".

Em [6] um estudo numérico foi realizado utilizando o método Constructal Design para otimizar uma aleta retangular inserida em uma cavidade retangular submetida ao escoamento do ar, em regime laminar e transferência de calor por convecção forçada. Na modelagem computacional foram utilizados os softwares GAMBIT e FLUENT. Dentre os principais resultados destaca-se que, à medida em que o número de Reynolds aumentou, a proporção analisada diminuiu substancialmente para aletas com menor penetração na cavidade.

Nesse contexto, outros trabalhos podem ser mencionados como formas bem-sucedidas de aplicação do método Constructal Design na otimização geométrica [7,8,9,10,11]. Aliado a isso, o (FEM - Finite Element Method) mostrou-se apropriado para o estudo numérico de otimização geométrica de materiais em forma de "Y" inseridos em corpos sólidos [12,13].

Portanto, o objetivo deste trabalho é a minimização da resistência térmica global, entre o sistema estudado e o meio externo, através da variação geométrica de um material de alta condutividade térmica em forma de "T" inserido em um sólido de baixa condutividade térmica empregando o método Constructal Design. Para isso, são mantidas constantes as quantidades dos materiais de alta condutividade e do sólido de baixa condutividade térmica.

Essa proposta de trabalho contribuiu com a geração de resultados preliminares que servirão de base para a continuidade deste estudo, onde serão comparadas diferentes propostas de condutividade térmica para a geometria em forma de " $\mathrm{T}$ " com a área total ocupada por esta geometria inserida no sólido de baixa condutividade térmica.

\section{MATERIAL E MÉTODOS}

Nesta seção será apresentada uma breve revisão bibliográfica sobre o método Constructal Design, a descrição física e geométrica do problema em estudo, a modelagem matemática e a modelagem numérica utilizada neste trabalho. 


\subsection{MÉTODO CONSTRUCTAL DESIGN}

A teoria Constructal explica como ocorre a organização, diversidade e complexidade de sistemas naturais onde existe alguma espécie de fluxo através do tempo e espaço, tais como: bacias hidrográficas, pulmões, circulação atmosférica, formas de animais, tecidos vascularizados entre outros. A teoria Constructal prima pela configuração do fluxo (geometria e arquitetura), fundamentada em um princípio de forma, geração e evolução no tempo na direção do maior acesso global do fluxo em sistemas que podem variar a geometria submetida às restrições [2,14].

A lei Consctrutal afirma que: "Para um sistema de dimensões finitas, onde existe fluxo (movimento), persistir no tempo (sobreviver) ele deve evoluir de uma maneira que propicie o mais fácil acesso possível às correntes que escoam através dele" $[1,14]$.

O método Constructal Design, por sua vez, é baseado na lei Constructal, que é um princípio físico fundamental. Dessa forma, o mesmo está sujeito às restrições e objetivos do sistema. Em engenharia, por exemplo, o referido método é amplamente aplicado à otimização geométrica de sistemas de fluxo. Isso ocorre principalmente em problemas relacionados à transferência do calor e à mecânica dos fluidos [14].

Portanto, os projetos de engenharia, dentre outros aspectos, caracterizam-se pela configuração, a qual sendo livre para variar, faz com que o desempenho do sistema tenha condições de alcançar melhorias. Assim, o esforço para melhorar o sistema pode ser compreendido como o princípio Constructal da ótima distribuição das imperfeições. Essa distribuição diminui as irreversibilidades, a fim de minimizar a quantidade de energia útil perdida pelo sistema [14].

\subsection{DESCRIÇÃO DO PROBLEMA}

Primeiramente, considere um material sólido que constitui uma placa (PI), idealizada, componente de um determinado circuito eletrônico (Figura 1).

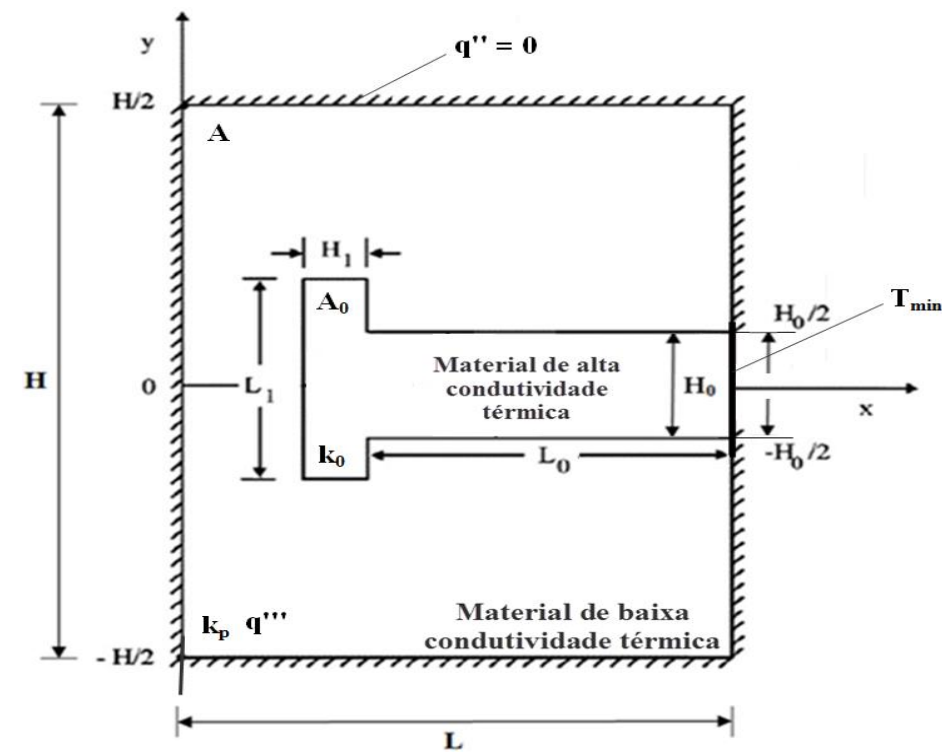

Figura 1: Geometria do corpo sólido com baixa condutividade e com geração de calor com vias em formato de " $T$ " de alta condutividade.

A mesma pode ser representada como uma placa onde há geração de calor uniforme em cada ponto da geometria do material, representado pela taxa de geração de calor volumétrica $\left(q^{\prime \prime \prime}\right)$ $\left[\mathrm{W} / \mathrm{m}^{3}\right]$, que simula o calor gerado pelo funcionamento dos componentes eletrônicos. O material (PI) é considerado isotrópico e com condutividade térmica $\left(k_{p}\right)[\mathrm{W} /(\mathrm{m} \cdot \mathrm{K})]$ constante. Para simplificar o estudo, a geometria é assumida bidimensional, com a hipótese da terceira dimensão $(W)$, espessura, ser suficientemente longa em comparação com as outras duas 
dimensões, a altura $(H)$ e o comprimento $(L)[\mathrm{m}]$. Essas duas últimas dimensões definem a área superficial $(A)$, em $\left[\mathrm{m}^{2}\right]$, do referido material. O problema se fundamenta na condição térmica de que a placa (PI) não pode atingir uma determinada temperatura, pois esta situação causaria dano e/ou falha nos componentes eletrônicos. Uma solução para este problema consiste em retirar calor da placa por meio de materiais de elevada condutividade (principalmente em sistemas sem espaço para inserção de sistemas de refrigeração por convecção).

Isso seria possível, colocando uma quantidade limitada de um determinado material metálico de alta condutividade $(\mathrm{MC})$, com condutividade térmica $\left(k_{0}\right),[\mathrm{W} /(\mathrm{m} \cdot \mathrm{K})]$, sendo: $\left(k_{0}>k_{p}\right)$. O material (MC) segue uma configuração geométrica em forma de "T", com área definida por $\left(A_{0}\right)$ $\left[\mathrm{m}^{2}\right]$. A base primária ou principal do referido material é definida por $\left(H_{0}\right)[\mathrm{m}]$ e o comprimento principal é dado por $\left(L_{0}\right)$ [m]. A base secundária é dada por $\left(L_{1}\right)$ [m] e o comprimento secundário é definido por $\left(H_{l}\right)[\mathrm{m}]$. As paredes externas do material $(\mathrm{PI})$ foram consideradas isoladas termicamente $\left(q^{\prime \prime}=0\right)$ (condição de contorno de Neumann ou de segunda espécie). Na parede representada por $\left(H_{0}\right)$ foi imposta a condição de temperatura prescrita $\left(T_{\min }\right)[\mathrm{K}]$ (condição de contorno de Dirichlet ou de primeira espécie) (Figura 1). A taxa de geração de energia gerada $\left(q^{\prime \prime \prime} A W\right)$ foi removida por dissipadores de calor localizados na borda do corpo (meio externo) à temperatura $\left(T_{\min }\right)$.

Dessa forma, a finalidade deste trabalho é a minimização da resistência térmica global, $\left(T_{\max }-T_{\min }\right) /\left(q^{\prime \prime \prime} A\right)$, entre o sistema estudado e o meio externo, através da variação na geometria de um material de alta condutividade térmica em forma de "T" inserido em um sólido de baixa condutividade térmica empregando o método Constructal Design. No entanto, no decorrer da implementação, são mantidas constantes as quantidades dos materiais de alta condutividade e do sólido de baixa condutividade térmica.

\subsection{MODELAGEM MATEMÁTICA}

Esta abordagem se fundamenta no princípio físico da conservação da energia. Dessa forma, o modelo matemático para a difusão do calor, em regime permanente, com geração de calor uniforme, propriedades termofísicas constantes, abordagem bi-dimensional, é dado por $[15,16]$ :

$$
\frac{\partial^{2} T}{\partial x^{2}}+\frac{\partial^{2} T}{\partial y^{2}}+\frac{q^{\prime \prime \prime}}{k_{p}}=0
$$

A Equação (1) pode ser adimensionalizada usando os seguintes grupos adimensionais $[4,8,9,10]$ :

$$
\theta=\frac{T-T_{\min }}{q^{\prime \prime \prime} A / k_{p}} ; \tilde{k}=\frac{k_{0}}{k_{p}} ; \tilde{x}, \tilde{y}, \widetilde{H}_{0}, \widetilde{L}_{0}, \widetilde{H}, \widetilde{L}, \widetilde{H}_{1}, \widetilde{L}_{1}=\frac{x, y, H_{0}, L_{0}, H, L, H_{1}, L_{1}}{A^{1 / 2}}
$$

onde: $T$ é a temperatura máxima do componente $[\mathrm{K}]$, de tal forma que, quanto mais elevado é o valor de $T$, menor será a segurança do equipamento. Neste trabalho foi utilizado $\tilde{k}=100$, sendo que: $k_{0}=100,0 \mathrm{~W} /(\mathrm{m} \cdot \mathrm{K})$ e $k_{p}=1,0 \mathrm{~W} /(\mathrm{m} \cdot \mathrm{K})$.

Portanto, a Equação (1) toma a forma adimensional que segue expressa por:

$$
\frac{\partial^{2} \theta}{\partial \tilde{x}^{2}}+\frac{\partial^{2} \theta}{\partial \tilde{y}^{2}}+1=0
$$

De forma semelhante, a equação da condução do calor também é resolvida para a região limitada pelo material de alta condutividade (MC). Nessa região é assumida a condição de que nenhum circuito (componente eletrônico) é colocado sobre o material (MC), de modo que não haja geração de calor na área ocupada por $\left(A_{0}\right)$ isto é, $\left(q^{\prime \prime \prime}=0\right)$. Dessa forma, a equação da condução de calor adimensional, para tal região, é dada por: 


$$
\frac{\partial^{2} \theta}{\partial \tilde{x}^{2}}+\frac{\partial^{2} \theta}{\partial \tilde{y}^{2}}=0
$$

A resistência térmica ou excesso de temperatura máxima pode ser expresso, na forma adimensional, por $[4,8,12,13]$ :

$$
\theta_{\max }=\frac{T_{\max }-T_{\min }}{q^{\prime \prime \prime} A / k_{p}}
$$

onde: $T_{\max }$ é a temperatura máxima do componente. A Equação (5), por conta da aplicação do método Constructal Design, passa a ser a função objetivo do problema.

Para aplicação do método Constructal Design, são consideradas constantes as áreas dos materiais (PI) e (MC), respectivamente, representados por $A$ e $A_{0}$ (restrições geométricas do problema) (Figura 1).

A área da placa (PI) é dada por:

$$
A=H L
$$

Em termos adimensionais, tem-se que:

$$
\frac{A}{A}=\frac{H L}{A^{1 / 2} A^{1 / 2}}
$$

Logo, tem-se, na forma adimensionalizada que:

$$
\widetilde{H} \tilde{L}=1
$$

A área ocupada pelo material (MC) é dada por:

$$
A_{0}=H_{0} L_{0}+H_{1} L_{1}
$$

A adimensionalização de $A_{0}$, é expressa através do parâmetro $\phi$ dado por:

$$
\phi=\frac{A_{0}}{A}=\frac{H_{0} L_{0}+H_{1} L_{1}}{A^{1 / 2}}
$$

Tem-se, então, que:

$$
\phi=\widetilde{H}_{0} \widetilde{L}_{0}+\widetilde{H}_{1} \widetilde{L}_{1}
$$

As demais variáveis do problema são apresentadas, resumidamente, na forma adimensional e são dadas por:

$$
\begin{aligned}
& \tilde{L}=\left[\frac{1}{(H / L)}\right]^{1 / 2} \\
& \widetilde{H}=\left(\frac{1}{\tilde{L}}\right) \\
& \tilde{L}_{1}=\frac{\phi}{\left[\left(H_{0} / L_{0}\right) \cdot\left(L_{0} / L_{1}\right)^{1 / 2}+2 \cdot\left(H_{1} / L_{1}\right)\right]^{1 / 2}} \\
& \tilde{L}_{0}=\left(\frac{L_{0}}{L_{1}}\right) \tilde{L}_{1}
\end{aligned}
$$




$$
\widetilde{H}_{0}=\left(\frac{H_{0}}{L_{0}}\right) \tilde{L}_{0}
$$

Para a placa (PI), a condição de contorno térmica de fluxo prescrito nulo foi imposta da seguinte forma:

$$
\begin{aligned}
& \frac{\partial T}{\partial x}=0, \text { para: } x=0 \mathrm{e}-\frac{H}{2} \leq y \leq \frac{H}{2} \text { e para: } x=L \mathrm{e}\left[\left(-\frac{H}{2} \leq y \leq-\frac{H_{0}}{2}\right) \cup\left(\frac{H_{0}}{2} \leq y \leq \frac{H}{2}\right)\right] \\
& \frac{\partial T}{\partial y}=0, \text { para: } y=-\frac{H}{2} \text { e } 0 \leq x \leq L \text { e para: } y=\frac{H}{2} e 0 \leq x \leq L
\end{aligned}
$$

Além disso, na placa (PI), também é imposta a condição de contorno térmica de temperatura prescrita, conforme segue:

$$
\text { Para: } x=L \mathrm{e}-\frac{H_{0}}{2} \leq y \leq \frac{H_{0}}{2} \text {, tem-se: } T=T_{\min }
$$

Com as geometrias determinadas através do método Constructal Design, a próxima etapa é obter a solução do campo de temperaturas para o domínio para todas as geometrias analisadas em função dos parâmetros conhecidos. Para isso, foi utilizada uma metodologia numérica.

\subsection{MODELAGEM NUMÉRICA}

O FEM consiste em um método numérico aproximado para análise de diversos fenômenos físicos que ocorrem em meios contínuos e que são descritos através de equações diferenciais parciais (EDP), com determinadas condições de contorno (problemas de valor de contorno) e possivelmente com condições iniciais (para problemas no regime transiente). O FEM é bastante genérico e pode ser aplicado na solução de inúmeros problemas da engenharia [17].

Neste trabalho, a ferramenta PDETOOL do software MATLAB foi utilizada para a solução de EDP, utilizando o FEM. Essa metodologia mostrou-se apropriada para problemas de transferência do calor por difusão térmica em trabalhos anteriores encontrados na literatura $[4,18]$.

A malha adotada é não-uniforme (elementos triangulares) e foi determinada por meio de refinamentos sucessivos e o critério utilizado para determinação da malha independente foi $\left|\left(\theta_{\max }^{j}-\theta_{\max }^{j+1}\right) / \theta_{\max }^{j}\right| \leq 5,0 \times 10^{-5}$, onde: $\theta_{\max }^{j}$ é a temperatura máxima em excesso (adimensional) calculada utilizando a quantidade de células da malha corrente e $\theta_{\max }^{j+1}$ é a temperatura máxima em excesso (adimensional) utilizando a malha seguinte, a qual o número de células foi aumentada em quatro vezes em relação à malha anterior. Portanto, a malha independente foi constituída de 22.464 células, conforme a Tabela 1.

A validação do modelo computacional utilizado neste trabalho pode ser encontrada em $[19,20]$ com riqueza de detalhes e não será aqui abordada pela brevidade e objetividade do texto. A partir dessa validação é possível a geração de novos resultados numéricos em diferentes aplacações.

\section{RESULTADOS E DISCUSSÃO}

Neste problema de otimização geométrica, tem-se 6 dimensões necessárias para construção do domínio computacional: $H, L, H_{1}, L_{1}, H_{0}$ e $L_{0}$. Para a construção das geometrias são considerados constantes os parâmetros $\phi=0,1$ e $H / L=1,0$. A aplicação do método Constructal Design se dá neste caso com a utilização de três graus de liberdade: $H_{1} / L_{1}, H_{0} / L_{0}$ e $L_{0} / L_{1}$. Para $H_{1} / L_{1}$ foram assumidos os seguintes valores: 0,$05 ; 0,01 ; 0,6$ e 1,0 . Para cada valor de $H_{1} / L_{1}$, foram definidos as seguintes possibilidades para $H_{0} / L_{0} ; 0,035 ; 0,1 ; 0,5$ e 1,0 . A fração $L_{0} / L_{1}$ variou livremente sujeita às restrições do problema definindo a forma das geometrias analisadas. 
A Figura 2, de forma ilustrativa, mostra o fluxograma do processo de otimização geométrica através do Constructal Design.

Tabela 1: Teste numérico mostrando a aquisição de uma malha independente.

$$
\left(\phi=0,1 ; H / L=1,0 ; H_{1} / L_{1}=0,6 ; H_{0} / L_{0}=0,5 ; L_{0} / L_{1}=0,5\right)
$$

\begin{tabular}{ccc}
\hline & \multicolumn{2}{c}{ independência de malha } \\
\cline { 2 - 3 } Número de elementos & $\boldsymbol{\theta}_{\text {max }}$ & $\left|\left(\boldsymbol{\theta}_{\text {max }}^{\boldsymbol{j}}-\boldsymbol{\theta}_{\text {max }}^{\boldsymbol{j}+\mathbf{1}}\right) / \boldsymbol{\theta}_{\text {max }}^{\boldsymbol{j}}\right|$ \\
\hline 351 & 0,373143 & $2,33 \times 10^{-2}$ \\
1.404 & 0,381846 & $9,53 \times 10^{-3}$ \\
5.616 & 0,385487 & $5,78 \times 10^{-4}$ \\
22.464 & 0,385710 & $2,07 \times 10^{-5}$ \\
89.856 & 0,385718 & -------- \\
\hline
\end{tabular}

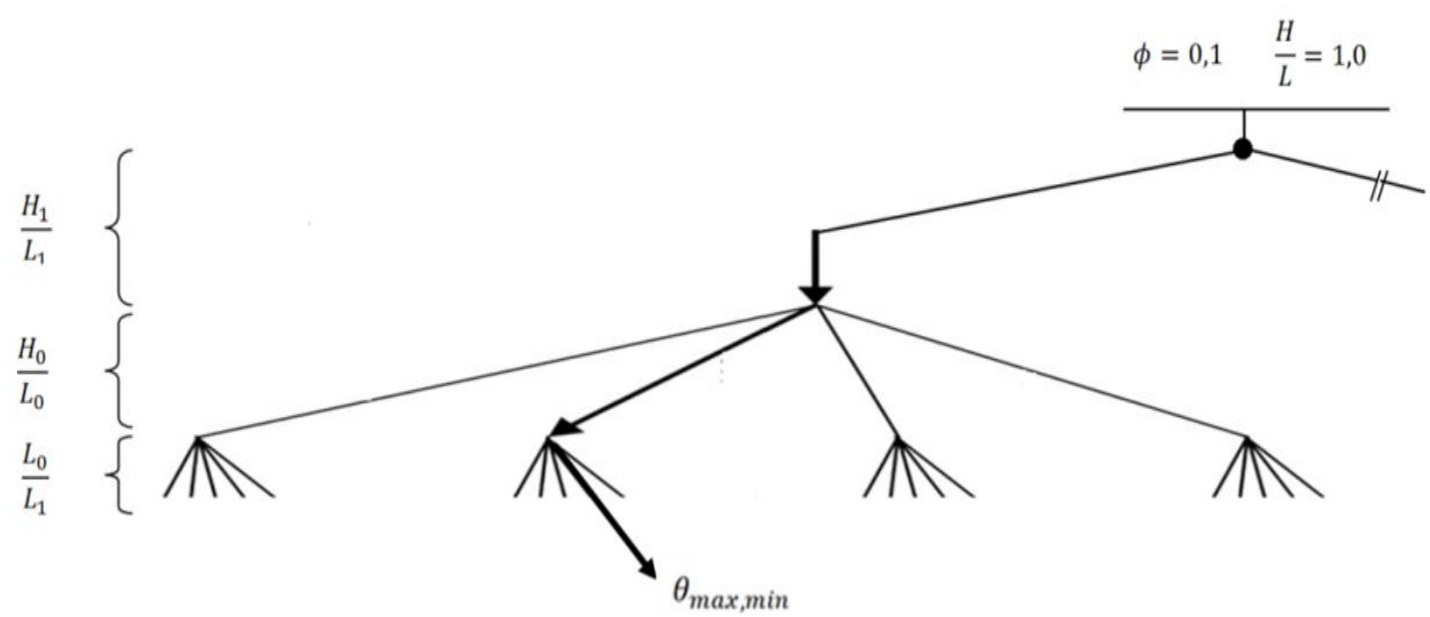

Figura 2: Fluxograma ilustrativo do processo de otimização geométrica através do Constructal Design.

A Figura 3 (a) mostra o efeito da razão $L_{0} / L_{1}$ sobre $\theta_{\max }$ para os valores de $H_{0} / L_{0}$, sendo possível observar que $\theta_{\max }$ tende a diminuir na medida que $L_{0} / L_{1}$ aumenta. Essa tendência é observada para todos os valores de $H_{0} / L_{0}$ analisados, exceto para $H_{0} / L_{0}=1,0$ onde $\theta_{\max }$ cresce novamente após alcançar a temperatura máxima em excesso adimensional uma vez minimizada, i.e., para $L_{0} / L_{1} \geq 0,574$. Com relação às geometrias ótimas alcançadas para a menor relação de $H_{0} / L_{0}=0,035$ a geometria ótima foi obtida no limite superior de $L_{0} / L_{1}$ investigado. Para $H_{0} / L_{0}=$ 0,1 a magnitude de $\theta_{\max }$ diminuiu na região de ótimo. Para maiores valores de $H_{0} / L_{0}(0,5$ e 1,0$)$ a magnitude de $\theta_{\max }$ cresce novamente para vários valores de $L_{0} / L_{1}$. Os resultados indicam que a alteração da razão $H_{0} / L_{0}$ afeta o efeito da razão $L_{0} / L_{1}$ sobre o desempenho térmico do problema estudado. Os resultados ótimos obtidos na Figura 3 (a) para várias razões de $H_{1} / L_{1}$ são sumarizados na Figura 3 (b), mais precisamente é apresentado o efeito da razão $H_{0} / L_{0}$ sobre $\theta_{\max \text {,min }}$ para várias razões de $H_{1} / L_{1}$. É possível notar um comportamento semelhante para as curvas formadas para os diferentes valores de $H_{1} / L_{1}$, com exceção da razão $H_{1} / L_{1}=0,01$ onde a geometria ótima foi obtida para a menor razão de $H_{0} / L_{0}$. Para todos os casos apresentados, a razão de $H_{0} / L_{0}$ foi obtida para $\left(H_{0} / L_{0}\right)_{\mathrm{o}}=0,1$. Para a razão $\left(H_{1} / L_{1}\right)_{\mathrm{o}}=0,01$ (uma vez otimizada) e $\left(H_{0} / L_{0}\right)_{\text {oо }}=0,1$ (duas vezes otimizadas) obtém-se a temperatura máxima em excesso adimensional duas vezes minimizada de $\theta_{\max , m m}=0,1285$. 


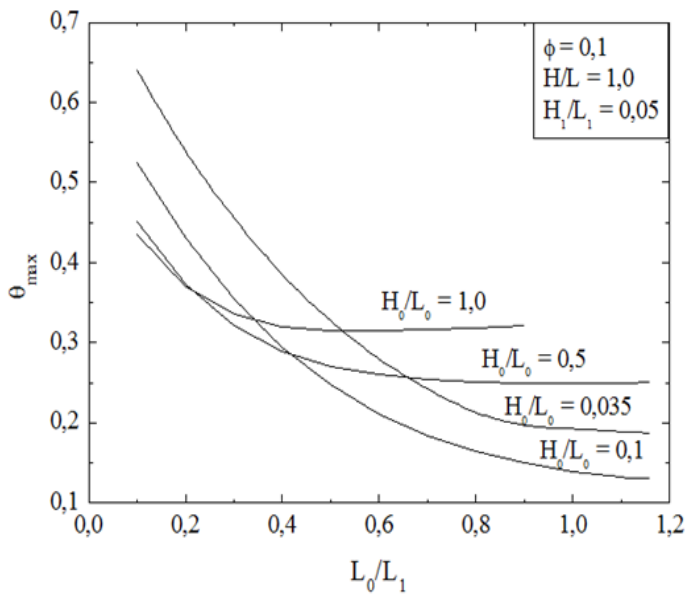

(a)

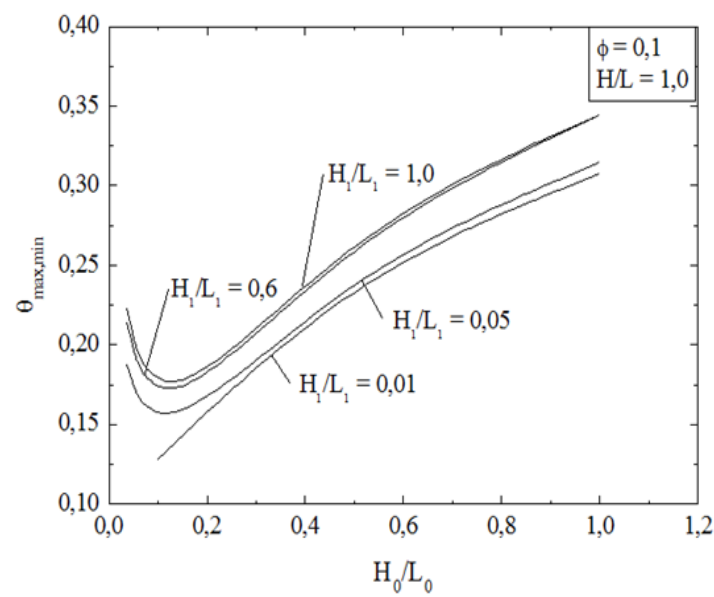

(b)

Figura 3: a) Efeito de $L_{0} / L_{1}$ sobre $\theta_{\max }$ para várias razões de $H_{0} / L_{0}$, b) Efeito de $H_{0} / L_{0}$ sobre $\theta_{\max \text {,min }}$ para várias razões de $H_{1} / L_{1}$.

Com relação ao efeito da razão $H_{0} / L_{0}$ sobre a razão $\left(L_{0} / L_{1}\right)_{\text {o }}$ para os diferentes valores de $H_{1} / L_{1}$ estudados, os resultados são apresentados na Figura 4 (a). Para os casos analisados foi possível observar um comportamento semelhante de $H_{0} / L_{0}$ sobre a razão $\left(L_{0} / L_{1}\right)_{0}$ para as maiores razões de $H_{1} / L_{1}$ estudados $\left(H_{1} / L_{1}=0,6\right.$ e 1,0) onde a razão $\left(L_{0} / L_{1}\right)_{0}$ cresce na faixa 0,01 $\leq H_{0} / L_{0} \leq 0,1$ e após essa faixa sofre uma redução intensa. Para as menores razões de $H_{1} / L_{1}$ é visto uma redução de $\left(L_{0} / L_{1}\right)_{\mathrm{o}}$ com o aumento de $H_{0} / L_{0}$, onde para a menor razão de $H_{1} / L_{1}=0,01$ a diminuição de $\left(L_{0} / L_{1}\right)_{\mathrm{o}}$ é quase linear. A partir dos resultados ótimos obtidos na Figura 4 (a) é possível avaliar o efeito da razão $H_{1} / L_{1}$ sobre a temperatura máxima em excesso adimensional das vezes minimizada, $\theta_{\max , m m}$, e suas respectivas geometrias ótimas, $\left(H_{0} / L_{0}\right)_{\mathrm{o}}$ e $\left(L_{0} / L_{1}\right)_{\mathrm{oo}}$. Os resultados desse efeito podem ser vistos na Figura 4 (b). Pode ser observado que as menores razões de $H_{1} / L_{1}$ conduzem ao melhor desempenho térmico do problema em análise, i.e., que reduzem $\theta_{\max , m m}$. Com relação ao efeito de $H_{1} / L_{1}$ sobre as geometrias ótimas, pode ser visto que a razão $\left(H_{0} / L_{0}\right)_{0}$ é praticamente insensível à variação da razão $H_{1} / L_{1}$, enquanto a razão $\left(L_{0} / L_{1}\right)_{\text {oo }}$ sofre uma grande variação do menor valor de $H_{1} / L_{1}$ estudado $\left(H_{1} / L_{1}=0,01\right)$ até o extremo superior de $H_{1} / L_{1}\left(H_{1} / L_{1}=1,0\right)$, i.e., nesse nível de otimização o parâmetro $\left(L_{0} / L_{1}\right)_{\mathrm{oo}}$ possui maior influência sobre o desempenho térmico do problema do que a razão $\left(H_{0} / L_{0}\right)_{\text {o }}$.

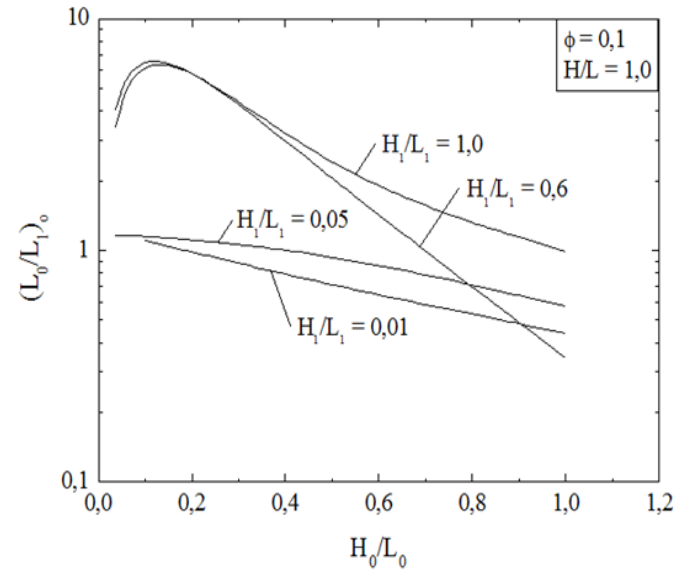

(a)

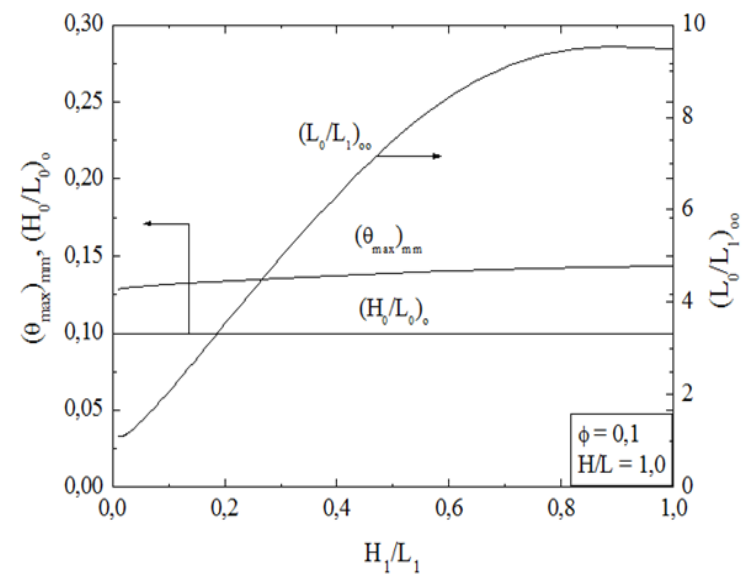

(b)

Figura 4: a) Efeito de $H_{0} / L_{0}$ sobre $\left(L_{0} / L_{1}\right)_{o}$ para várias razões de $H_{1} / L_{1}$, b) Efeito da razão $H_{1} / L_{1}$ sobre $\theta_{\max , m m}$ e suas respectivas geometrias ótimas. 
A Figura 5 (a) apresenta o domínio computacional discretizado em elementos finitos triangulares. Também é possível notar a configuração que a geometria em forma de "T" (material (MC)) exerce no sólido com geração de calor, material (PI). Essa forma assumida pelo material (MC) foi considerada a geometria ótima, pois proporcionou a menor resistência e isso ocorre quando a distribuição do campo de temperaturas é a mais homogênea, i.e., para os casos analisados essa geometria propiciou a melhor distribuição das imperfeições do sistema. Nesse sentido, a Figura 5 (b) traz o campo de temperaturas (adimensional) para a simulação numérica correspondente a geometria otimizada. É possível notar que o comprimento principal do material (MC), isto é, $\left(L_{0}\right)$ tomou grande progressão longitudinal (eixo x), assim como a base secundária $\left(L_{1}\right)$ também evoluiu no tamanho de forma transversal (eixo y), i.e., quando o caminho condutivo teve a maior penetração no domínio do sólido com geração de calor. As isotermas seguiram a tendência do material (MC) acarretando em pouco acúmulo de zonas com excessos de pontos quentes.

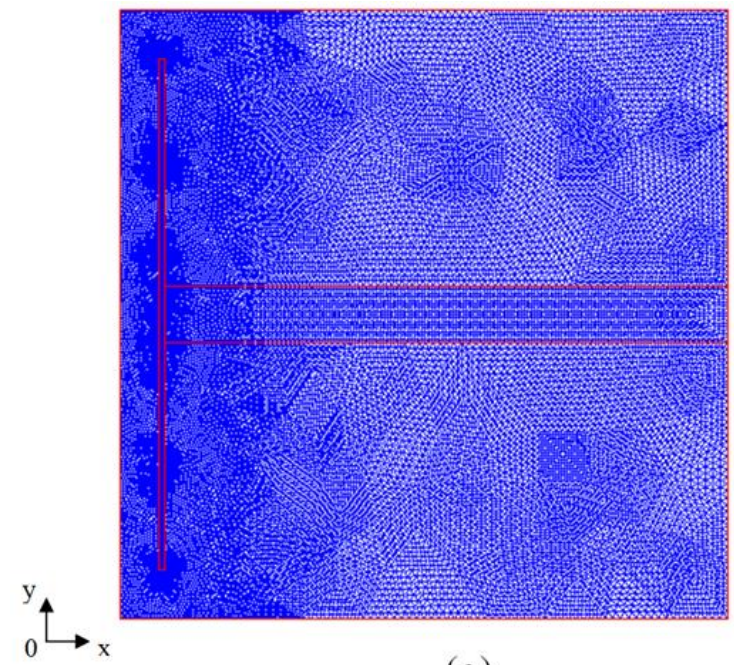

(a)

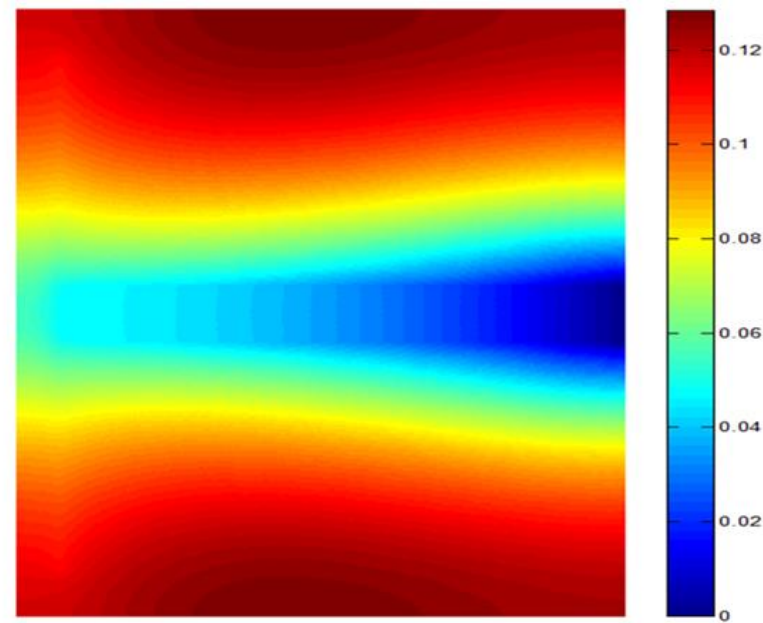

(b)

Figura 5: a) Domínio computacional discretizado, b) Campo de temperaturas para a geometria otimizada.

\section{CONCLUSÃO}

Por meio da aplicação do método Constructal Design, foi possível propor a minimização da resistência térmica global de um corpo sólido retangular com baixa condutividade e geração de calor com vias de alta condutividade em forma de "T" inseridas no domínio e ligadas em uma região externa de baixa temperatura (sumidouro). Para a avaliação geométrica desse problema foram mantidas constantes as áreas do sólido retangular e das vias de alta condutividade, mais precisamente foi considerada uma fração entre as áreas das vias e do sólido de $\phi=0,1$. No presente estudo também foi considerada fixa a razão $H / L=1,0$ e foram variados os graus de liberdade $H_{1} / L_{1}, H_{0} / L_{0}$ e $L_{0} / L_{1}$ permitindo minimizar três vezes a temperatura máxima em excesso adimensional $\left(\theta_{\max }\right)$.

Os resultados mostraram que para $\phi=0,1$ e $H / L=1,0$, a geometria três vezes otimizada foi encontrada para as razões $\left(H_{1} / L_{1}\right)_{\mathrm{o}}=0,01,\left(H_{0} / L_{0}\right)_{\mathrm{oo}}=0,1$ e $\left(L_{0} / L_{1}\right)_{\text {ooo }}=1,1$ e conduziram a uma $\theta_{\max , m m m}=0,1285$, o que é aproximadamente 5 vezes menor do que a pior geometria avaliada. Dessa forma, os resultados mostram a importância da avaliação geométrica para a melhora do desempenho térmico deste problema.

Além disso, os estudos mostram que a via de alta condutividade em forma de "T" tende a se inserir, longitudinalmente e transversalmente, de modo progressivo e evolutivo na placa (PI) buscando atingir as zonas mais distantes da parede do sólido onde a temperatura é prescrita $T_{m i n}$, i.e., a geometria do "T" com maior penetração nos sentidos longitudinal e transversal. Também 
foi possível observar que algumas razões geométricas possuem maior influência sobre o desempenho térmico do problema em relação a outras. Por exemplo, na avaliação do efeito de $\mathrm{H}_{1} / \mathrm{L}_{1}$ sobre as geometrias ótimas do "T", pode ser visto que a razão $\left(H_{0} / L_{0}\right)_{\mathrm{o}}$ é praticamente insensível à variação da razão $H_{1} / L_{1}$, enquanto a razão $\left(L_{0} / L_{1}\right)_{\mathrm{oo}}$ sofre uma grande variação do menor valor de $H_{1} / L_{1}$ estudado $\left(H_{1} / L_{1}=0,01\right)$ até o extremo superior de $H_{1} / L_{1}\left(H_{1} / L_{1}=1,0\right)$, i.e., nesse nível de otimização o parâmetro $\left(L_{0} / L_{1}\right)_{\text {oo }}$ possui maior influência sobre o desempenho térmico do problema do que a razão $\left(H_{0} / L_{0}\right)_{\text {。 }}$.

\section{AGRADECIMENTOS}

Os autores agradecem ao CNPq pelo apoio.

\section{REFERÊNCIAS BIBLIOGRÁFICAS}

1. Bejan A. Constructal-theory network of conducting paths for cooling a heat generating volume. Journal Heat MassTransfer. 1996;40(4):799-816.

2. Bejan A. Shape and Structure, from Engineering to Nature. Cambridge University Press, Cambridge, UK. 2000.

3. Eslami M, Jafarpur K. Thermal resistence in conductive constructal design of arbitrary configuration: A new general Approach. Energy Conversion and management. 2012;57:117-124.

4. Horbach C. Constructal design de materiais de alta condutividade em forma de "Y" para refrigeração de corpo gerador de calor. Dissertação de Mestrado, Universidade Federal do Rio Grande do Sul (UFRGS), Programa de Pós-Graduação em Engenharia Mecânica (PROMEC). 2013.

5. Lorenzini G, Biserni C, Rocha LAO. Constructal design of X-shaped condutive pathways for cooling a heat-generating body. International Journal of Heat and Mass Transfer. 2013;58:513-520.

6. Dos Santos ED, Isoldi LA, Goulart MM, Rodrigues MK, Seibt FM, Souza RV, Rocha LAO. Constructal design of a rectangular fin intruded into forced convective lid-driven cavity flows. Constructal Law Conference. Nanjing, China. Proceedings of Constructal Law Conference. 2013. p.126-134.

7. Bejan A, Dan N. Constructal trees of convective fins. Jounal of Heat and Mass Transfer. 1999;121: 675-682.

8. Bejan A, Almogbel M. Constructal T-shaped fins. International Journal of Heat and Mass Transfer. 2000;43:2101-2115.

9. Biserni C, Rocha LAO, Bejan A. Inverted fins: geometric optimization of the intrusion into a conducting wall. International Journal of Heat and Mass Transfer. 2004;47:2577-2586.

10. Bejan A, Rocha LAO, Lorente S. Thermodynamic optimization of geometry: T- and Y-shaped constructs of fluid streams. International Journal of Thermal Sciences. 2000;39:949-960.

11. Biserni C, Rocha LAO, Stanescu G, Lorenzini E. Constructal H-shaped cavities according to Bejan's theory. International Journal of Heat and Mass Transfer. 2007;50:2132-2138.

12. Lorenzini G, Rocha LAO. Constructal design of T-Y assembly of fins for an optimized heat removal. International Journal of Heat and mass Transfer. 2009;52:1458-1463.

13. Xie Z, Chen L, Sun F. Constructal optimization on T-shaped cavity based on entransy dissipation minimization. Chinese Science Bulletin. 2009;54:4418-4427.

14. Bejan A, Lorente S. Design With Constructal Theory. Wiley, Hoboken. 2008.

15. Maliska C. Transferência de calor e mecânica dos fluidos computacional. Livros Técnicos e Científicos Editora S.A., Rio de Janeiro. 2004. 453 p.

16. Incropera F, Dewitt D, Bergman T, Lavine A. Fundamentos de transferência de calor e de massa. $6^{\mathrm{a}}$ ed. Rio de Janeiro: LTC. 2008.

17. Lorenzini G, Biserni C, Link B., Isoldi L, dos Santos L, Rocha LAO. Constructal design of T-shaped cavity for several convective fluxes imposed at the cavity surfaces. Journal of Engineering Thermophysics. 2013;22:309-321.

18. Burnett D. Finite Element Analysis, from concepts to applications. Addison-Wesley. 1988.

19. Almogbel M, Bejan A. Conduction trees with spacing at tips. International Journal Heat Mass Transfer. 1999;42:3739-3756.

20.Lorenzini G, Biserni C, Rocha LAO. Constructal design of X-shaped conductive pathways for cooling a heat-generating body. International Journal of Heat and Mass Transfer. 2013;58:513-5208. 